Article

\title{
Research on Rotary Parts Vibration Suppression Based on Coaxiality Measurement and Unbalance Constraint
}

\author{
Yongmeng Liu ${ }^{1,2}$, Ruirui Li ${ }^{1,2}{ }^{(D}$, Chuanzhi Sun ${ }^{1,2, *}$, Ze Chen ${ }^{1,2}$, Yingjie Mei ${ }^{1,2} \mathbb{D}^{\text {, Pinghuan Xiao }}{ }^{1,2}$, \\ Xiaoming Wang ${ }^{1,2, *}$ and Chengtian $\mathrm{Li}^{3}$ \\ 1 Center of Ultra-Precision Optoelectronic Instrument Engineering, Harbin Institute of Technology, \\ Harbin 150080, China; lym@hit.edu.cn (Y.L.); 19B901021@stu.hit.edu.cn (R.L.); \\ 20s001065@stu.hit.edu.cn (Z.C.); 20B901021@stu.hit.edu.cn (Y.M.); 19S101141@stu.hit.edu.cn (P.X.) \\ 2 Key Lab of Ultra-Precision Intelligent Instrumentation Engineering (Harbin Institute of Technology), \\ Ministry of Industry and Information Technology, Harbin 150080, China \\ 3 Reactor Operation and Application Sub-Institute of Nuclear Power Institute of China, \\ Chengdu 610041, China; 19B908033@stu.hit.edu.cn \\ * Correspondence: czsun@hit.edu.cn (C.S.); wang_xm@hit.edu.cn (X.W.)
}

Citation: Liu, Y.; Li, R.; Sun, C.; Chen, Z.; Mei, Y.; Xiao, P.; Wang, X.; Li, C. Research on Rotary Parts Vibration Suppression Based on Coaxiality Measurement and Unbalance Constraint. Appl. Sci. 2021, 11, 5747. https://doi.org/10.3390/ app11125747

Received: 30 April 2021

Accepted: 18 June 2021

Published: 21 June 2021

Publisher's Note: MDPI stays neutral with regard to jurisdictional claims in published maps and institutional affiliations.

Copyright: (c) 2021 by the authors. Licensee MDPI, Basel, Switzerland. This article is an open access article distributed under the terms and conditions of the Creative Commons Attribution (CC BY) license (https:/ / creativecommons.org/licenses/by/ $4.0 /)$.

\begin{abstract}
To suppress the vibration of rotary parts, this paper established an unbalanced vibration response control model of rotary parts based on rotating axis coordinate system. This model considered the stacking transformation of geometric parameter errors and mass parameter errors of single stage rotor. First of all, the centroid transfer model based on the actual rotation axis was established, and the unbalanced excitation force vector of each stage of the rotor was studied. Secondly, the unbalanced excitation force vector of each stage of the rotor is substituted into the model of assembly vibration control based on the double constraints optimization strategy. Finally, the simulation analysis and the vibration experiment of three-stage rotor stacking assembly is carried out. The results show that the vibration of the engine rotor can be effectively suppressed by adjusting the assembly phase of the rotors, and the vibration amplitude of the combined rotor assembled by the double constraint optimization assembly strategy is $22.5 \%$ less than the vibration amplitude assembled by the direct assembly strategy. Besides, the coaxiality and the unbalance are reduced by $44.1 \%$ and $78.4 \%$, which fully shows the advantages of the double constraint optimization assembly strategy.
\end{abstract}

Keywords: rotary parts; centroid coordinate transformation; double constraint; assembly phase; vibration suppression

\section{Introduction}

The unbalance exciting force caused by the centroid deviation from the actual rotation axis when assembling is an important reason of the rotary parts vibration. Vibration will not only greatly influence engine performance, but also causes engine failure. To reduce vibration of the rotary parts effectively, and to improve the working efficiency and reliability of the rotor system and ensure the safety and stable operation of the rotary parts, the vibration suppression of the combined rotor by adjusting the unbalanced excitation force of the rotor can be a key solution [1-4].

To study the effects of rotor assembly on rotary parts performance, many scholars have done research on stacking methods and errors of rotary parts rotor [5-8]. S. S. Rao et al. [9] proposed a method of multi-level component assembly tolerance allocation and established the optimization model of assembly quality under the constraints of assembly precision and assembly function according to the requirements of tolerance allocation in assembly design process. T. Hussain et al. $[10,11]$ proposed three optimal assembly methods to optimize the geometric eccentricity of industrial turbine rotor and aircraft gas turbine rotor. Sun Chuanzhi et al. [12] analyzed the cumulative process of error transformation in the assembly process of multi-stage rotors and obtained the cumulative eccentricity error 
of each stage after multi-stage rotors assembly by using space vector projection method. Liu Jun et al. [13] proposed a technique to reduce the problem of rotor misalignment by combining the two-objective optimization of rotor stacking coaxiality and unbalance and studied a method to predict the geometric deviation between the rotating axis and its own centroid axis based on the error measurement of single-stage parts.

To improve the rotary parts performance and reduce vibration, more and more researches begin to study vibration suppression [14-16]. A. W Lees et al. [17] described the methods of rotor fault identification in recent decades, and the results shows that modelbased identification has played a significant role in the rapid resolution and quantification of faults. Zhou Shiyu et al. [18] developed a speed-varying transient rigid rotor model and can be used in the active vibration control or active balancing schemes for a rigid rotor. Yao Jianfei et al. [19] proposed two methods for the identification of the unbalance characteristics, and this technology can identify the axial location of the unbalance, its magnitude and phase. G. Chen [20] established a new dynamic model for a practical aero-engine which can effectively perform nonlinear dynamic simulations of a whole aero-engine with faults. Łukasz Bre'nkacz et al. [21,22] studied and discussed the effectiveness of vibration damping and proposed a method of vibration measurement by vision. Yao et al. [23] proposed a method to suppress the lateral vibration of rotor system by using grounded nonlinear energy receiver. Jiang Shuyun et al. [24] studied the suppression methods of lowfrequency vibration of flywheel energy storage system rotor bearing system, developed the radial magnetic pendulum tuned mass damper and axial magnetic pendulum tuned mass damper to reduce vibration. Lusty et al. [25] realized the active vibration control of flexible rotor by using the active magnetic brake with flexible inner stator and proposed an experimental device in line with the topology.

Scholars at home and abroad have made remarkable achievements in vibration control of rotor system, and active control methods of rotor vibration are gradually applied. However, the influence of the increase of exciting force caused by errors in assembly process on the vibration of the rotor system is ignored. In this paper, a vibration control method based on coaxiality and unbalance constraints is proposed. The calculation model of vibration and unbalance excitation force is established by using the mass transfer meter based on the actual rotation axis. The center of mass is the real center of mass coordinate of the rotor, and the axis of rotation is the geometric center line between the drive end and the non-drive end. The coaxiality and unbalance are constrained by adjusting the installation phase of rotary parts to reduce the unbalanced exciting force and achieve vibration suppression.

\section{Theoretical Model}

\subsection{Dynamic Analysis of Rotor Vibration Response Based on Centroid Transformation}

As shown in Figure 1, the reference axis of the traditional stacking assembly method is set through the center $O$ of the rotor bottom face and perpendicular to the bottom face. However, the center line of bearing at both ends of multistage rotor is taken as the actual rotation axis when doing vibration analysis of rotary parts rotor system. That is to say, the actual rotation axis $O O_{n A}$ is the connection line of the center of bottom face and the center of top face which deviates from the reference axis.

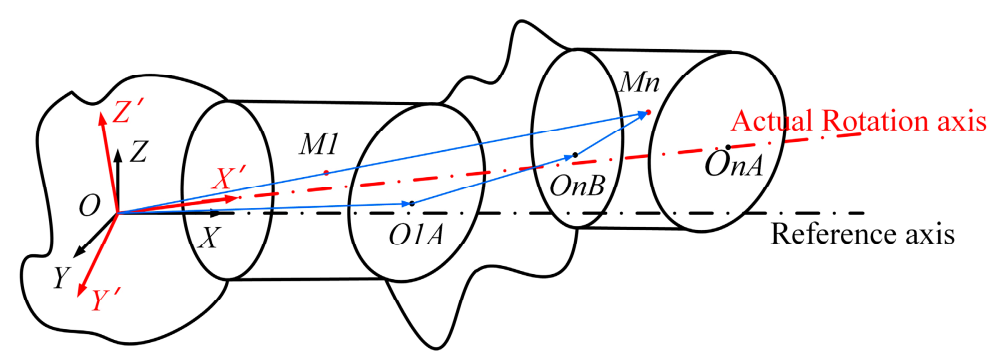

Figure 1. Multistage rotor centroid vector transformation model. 
The position vector of $k_{t h}$ rotor centroid deviation can be expressed as follows in a XOYZ coordinate system [26]:

$$
\boldsymbol{d} \boldsymbol{p}_{0-k}^{c}=\left[\begin{array}{c}
d x_{0-k}^{c} \\
d y_{0-k}^{c} \\
d z_{0-k}^{c}
\end{array}\right]=\sum_{i=1}^{k-1}\left(\prod_{j=1}^{i-1}\left(d \boldsymbol{R}_{j}^{r} d \boldsymbol{R}_{j}^{o}\right) d \boldsymbol{R}_{i}^{r} d p_{i}^{o}\right)+\prod_{i=1}^{k-1}\left(d R_{i}^{r} d R_{i}^{o}\right) d R_{k}^{r} d p_{k}^{c}
$$

where, $d x_{0-k}^{c}, d y_{0-k}^{c}, d z_{0-k}^{c}$ are the accumulated centroid deviation of $k_{t h}$ stage rotor in $X, Y, Z$ direction. $\boldsymbol{d} \boldsymbol{R}_{i}^{r}$ is the rotation matrix of $i_{t h}$ stage rotor and can be expressed as:

$$
\boldsymbol{d R}_{i}^{r}=\left[\begin{array}{ccc}
\cos \theta_{r i} & -\sin \theta_{r i} & 0 \\
\sin \theta_{r i} & \cos \theta_{r i} & 0 \\
0 & 0 & 1
\end{array}\right]
$$

where, $\theta_{r i}$ is the rotation angle of the $i_{t h}$ stage rotor around the reference axis.

$d R_{i}^{o}$ is the geometric eccentricity deviation matrix of $i_{t h}$ stage rotor and can be expressed as:

$$
\boldsymbol{d R}_{i}^{o}=\left[\begin{array}{ccc}
1 & 0 & \sin \theta_{t i} \cos \theta_{l i} \\
0 & 1 & \sin \theta_{t i} \sin \theta_{l i} \\
-\sin \theta_{t i} \cos \theta_{l i} & -\sin \theta_{t i} \sin \theta_{l i} & 1
\end{array}\right]
$$

where, $\theta_{t i}$ is the tilt angle of the assembly surface relative to the reference plane, and $\theta_{l i}$ is the position of the tilt angle on the assembly surface. $d p_{i}^{o}$ is the geometric center position vector matrix of $i_{\text {th }}$ stage rotor and can be expressed as:

$$
\boldsymbol{d} \boldsymbol{p}_{i}^{o}=\left[\begin{array}{l}
d x_{i}^{o} \\
d y_{i}^{o} \\
d z_{i}^{o}
\end{array}\right]
$$

where, $d x_{i}^{o}, d y_{i}^{o}, d z_{i}^{o}$ are the position vectors of the $i_{t h}$ rotor top face center in XOYZ coordinate system. $d p_{i}^{c}$ is the position vector matrix of the mass centroid of the $i_{t h}$ stage rotor, and can be expressed as:

$$
\boldsymbol{d} \boldsymbol{p}_{i}^{c}=\left[\begin{array}{l}
d x_{i}^{c} \\
d y_{i}^{c} \\
d z_{i}^{c}
\end{array}\right]
$$

where, $d x_{i}^{c}, d y_{i}^{c}, d z_{i}^{c}$ are the position vectors of the $i_{\text {th }}$ rotor mass centroid in XOYZ coordinate system. The position vector of the measuring surface center of the $k_{t h}$ stage rotor after assembly in the coordinate system XOYZ can be expressed as:

$$
d p_{0-k}^{o}=\left[\begin{array}{c}
d x_{0-k}^{o} \\
d y_{0-k}^{o} \\
d z_{0-k}^{o}
\end{array}\right]=\sum_{i=1}^{k}\left(\prod_{j=2}^{i}\left(d R_{j-1}^{r} d R_{j-1}^{o}\right) d R_{i}^{r} d p_{i}^{o}\right)
$$

The coordinate system $X^{\prime} O Y^{\prime} Z^{\prime}$ can be obtained through the rotation of the coordinate system XOYZ. Set the rotation axis direction vector as $l=\left(l_{x}, l_{y}, l_{z}\right)^{T}$. The unit vector of $l$ can be expressed as: $w\left(w_{x}, w_{y}, w_{z}\right)^{T}=\frac{l}{|l|}$. The rotation axis direction vector $l$ and the rotation angle $\theta$ can be obtained from Equations (6)-(8).

The position vector of the rotor top face center in the coordinate system XOYZ can be expressed as $\boldsymbol{P}\left(O_{n A x}, O_{n A y}, O_{n A z}\right)^{T}$. The position vector of the rotor top face center in the coordinate system $X^{\prime} O Y^{\prime} Z^{\prime}$ can be expressed as $Q\left(0,0, O_{n A z}\right)^{T}$, where, $O_{n A z}{ }^{\prime}=$ 
$\sqrt{\left(O_{n A x}\right)^{2}+\left(O_{n A y}\right)^{2}+\left(O_{n A z}\right)^{2}}$ according to the geometric relationship. The rotation axis direction vector $l$ and rotation angle $\theta$ are shown in Equations (7) and (8)

$$
\begin{gathered}
\boldsymbol{l}=\boldsymbol{P} \times \boldsymbol{Q}=\left[\begin{array}{c}
O_{n A y} O_{n A z^{\prime}} \\
-O_{n A x} O_{n A z^{\prime}} \\
0
\end{array}\right] \\
\theta=\arccos \left(\frac{\boldsymbol{P} \cdot \boldsymbol{Q}}{|\boldsymbol{P} \| \boldsymbol{Q}|}\right)
\end{gathered}
$$

Set the mass centroid position vector of the $k_{t h}$ rotor as $\left(d x_{0-k}^{\prime c}, d y_{0-k}^{\prime c}, d z_{0-k}^{\prime c}\right)^{\mathrm{T}}$, and it can be expressed in the $X^{\prime} O Y^{\prime} Z^{\prime}$ coordinate system:

$$
\left[\begin{array}{l}
d x^{\prime c} \\
d y_{0-k}^{\prime c} \\
d z_{0-k}^{\prime c}
\end{array}\right]=A\left[\begin{array}{l}
d x_{0-k}^{c} \\
d y_{0-k}^{c} \\
d z_{0-k}^{c}
\end{array}\right]
$$

where, $A$ is the rotation matrix which can transfer coordinate system $X O Y Z$ to coordinate system $O X^{\prime} Y^{\prime} Z^{\prime}$ and can be expressed as follows:

$$
\boldsymbol{A}=\left[\begin{array}{ccc}
\cos \theta+w_{x}^{2}(1-\cos \theta) & w_{x} w_{y}(1-\cos \theta)-w_{z} \sin \theta & w_{y} \sin \theta+w_{x} w_{z}(1-\cos \theta) \\
w_{z} \sin \theta+w_{x} w_{y}(1-\cos \theta) & \cos \theta+w_{y}^{2}(1-\cos \theta) & -w_{x} \sin \theta+w_{y} w_{z}(1-\cos \theta) \\
-w_{y} \sin \theta+w_{x} w_{z}(1-\cos \theta) & w_{x} \sin \theta+w_{y} w_{z}(1-\cos \theta) & \cos \theta+w_{z}^{2}(1-\cos \theta)
\end{array}\right]
$$

where, $\theta=\arccos \left(\frac{P \cdot Q}{|P||Q|}\right) \cdot Q^{r}$ is the unbalanced exciting force of rotor in the $X^{\prime} O Y^{\prime} Z^{\prime}$ coordinate system, and can be expressed as Equation (11) when the rotation speed of the rotor is $\Omega$.

$$
Q^{r}=m_{k} \Omega^{2}\left[\begin{array}{c}
d x_{0-k}^{\prime c} \\
d y_{0-k}^{\prime c} \\
0 \\
0
\end{array}\right] \cos \Omega t+m_{k} \Omega^{2}\left[\begin{array}{c}
-d y^{\prime c} \\
d x_{0-k}^{\prime c k} \\
0 \\
0
\end{array}\right] \sin \Omega t
$$

The unbalanced force of the system can be expressed as:

$$
Q^{r}=Q_{c} \cos \Omega t+Q_{s} \sin \Omega t
$$

Then the steady solution of the motion differential equation of the rotor system can be assumed to be:

$$
\boldsymbol{q}=\boldsymbol{q}_{c} \cos \Omega t+\boldsymbol{q}_{s} \sin \Omega t
$$

The steady motion equation of rotor system is shown as follows:

$$
M \ddot{q}+(C-\Omega G) \dot{q}+K \boldsymbol{q}=Q
$$

where $M$ is the mass assembly matrix which contains the translational and rotary effects of the shaft element and the disk element. Just like $M, G$ is the gyroscopic assembly matrix which considers the effects of the shaft element and the disk element. $C$ is the damping assembly matrix. $K$ is the stiffness assembly matrix which considers stiffness effects of the shaft element and the disk element. $Q$ is the excitation matrix.

To study the influence of unbalanced exciting force caused by centroid offset on a rotor system, $Q$ is the unbalanced exciting force which is only considering the unbalanced force caused by multi-stage rotors stacking. We can get the following results by substituting Equation (13) into Equation (14):

$$
\left[\begin{array}{l}
\boldsymbol{q}_{c} \\
\boldsymbol{q}_{s}
\end{array}\right]=\left[\begin{array}{cc}
\boldsymbol{K}-\boldsymbol{M} \Omega^{2} & \Omega(\boldsymbol{C}-\Omega G) \\
-\Omega(\boldsymbol{C}-\Omega \boldsymbol{G}) & \boldsymbol{K}-\boldsymbol{M} \Omega^{2}
\end{array}\right]^{-1}\left[\begin{array}{l}
\boldsymbol{Q}_{c} \\
\boldsymbol{Q}_{s}
\end{array}\right]
$$


The unbalance response is as following:

$$
\boldsymbol{q}=\boldsymbol{q}^{\prime} \cos (\Omega t-\varphi)
$$

where,

$$
\left\{\begin{array}{c}
\boldsymbol{q}^{\prime}=\sqrt{\boldsymbol{q}_{c}^{2}+\boldsymbol{q}_{s}^{2}} \\
\varphi=\arctan \frac{\boldsymbol{q}_{s}}{\boldsymbol{q}_{c}}
\end{array}\right.
$$

\subsection{Assembly Strategy Analysis}

The overall vibration of rotary parts multi-stage rotors is an important parameter to evaluate the assembly quality. The eligibility criteria of geometric parameters and mass characteristics of the rotary parts must be considered also. Different assembly methods in the assembly process of rotary parts rotor result in different centroid offset axis distance, which leads to different geometric error, mass error, and vibration of each stage rotor. Therefore, this paper proposes three assembly methods to optimize the vibration of engine rotor under the premise of considering the geometric error, mass error, and vibration suppression of rotary parts rotor. Three assembly strategies are as follows:

\subsubsection{First Assembly Strategy: Direct Assembly}

Take the assembly phase as the control variable valve and the vibration amplitude of the combined rotor under the action of unbalanced exciting force is as follows by using direct assembly:

$$
\left\{q_{\text {direct }}=\left\{\begin{array}{c}
\left.\sqrt{q_{c}^{2}\left(\theta_{r k}\right)+q_{s}^{2}\left(\theta_{r k}\right)}\right\} \quad k=1,2, \ldots n \\
\theta_{r k}=0
\end{array}\right.\right.
$$

where, $q_{\text {direct }}$ is the vibration amplitude of the rotary parts rotor under the first assembly strategy. $\theta_{r k}$ is the assembly phase of the $k_{t h}$ stage rotor. $q_{c}\left(\theta_{r k}\right)$ and $q_{s}\left(\theta_{r k}\right)$ are the two vibration amplitudes of the rotor in the orthogonal direction of the actual rotation axis.

\subsubsection{Second Assembly Strategy: Minimize Vibration Assembly}

The vibration amplitude of the combined rotor by using the second assembly strategy under the action of unbalanced exciting force is as follows:

$$
\left\{\begin{array}{c}
q_{\min }=\min \left\{\sqrt{q_{c}^{2}\left(\theta_{r k}\right)+q_{s}^{2}\left(\theta_{r k}\right)}\right\} \quad k=1,2, \ldots n \\
\theta_{r k}=0, \frac{360}{t_{r k}}, \ldots, 360-\frac{360}{t_{r k}}
\end{array} \quad k\right.
$$

where, $q_{\min }$ is the minimum vibration amplitude after rotor assembled by the second strategy, and $t_{r k}$ is the number of bolt holes on the assembly surface of the $k_{t h}$ rotary parts rotor.

\subsubsection{Third Assembly Strategy: Optimal Assembly with Double Constraints}

To minimize the vibration of the rotor, and to limit the unbalance and coaxiality valve of the multistage rotors, the optimal assembly strategy with double constraints is carried out and is shown as:

$$
\left\{\begin{array}{c}
q_{\text {double }}=\min \left\{\sqrt{q_{c}^{2}\left(\theta_{r k}\right)+q_{s}^{2}\left(\theta_{r k}\right)}\right\} \\
c\left(\theta_{r k}\right) \leq c_{\text {nlimit }} \\
u\left(\theta_{r k}\right) \leq u_{n l i m i t} \\
\theta_{r k}=0, \frac{360}{t_{r k}}, \ldots, 360-\frac{360}{t_{r k}}
\end{array}\right.
$$

where $q_{\text {doule }}$ is the vibration amplitude assembled by the third assembly strategy with the constraints of coaxiality and unbalance. $c\left(\theta_{r k}\right)$ represents the coaxiality of $k_{t h}$ stage rotor, and $c_{n l i m i t}$ represents the maximum coaxiality allowed after assembly of $k_{t h}$ stage 
rotor. $u\left(\theta_{r k}\right)$ represents the unbalance of $k_{t h}$ stage rotor, and $u_{n l i m i t}$ represents the maximum unbalance allowed after assembly of $k_{t h}$ stage rotor, respectively.

\section{Simulation}

To study the vibration suppression effect of this dynamic analysis model of unbalanced vibration response model based on centroid stacking transformation, the dynamic simulation analysis of three-stage rotors stacking assembly is carried out. The finite element method was used for analysis. This method simplifies the rotor system into discrete rigid, elastic shaft segments, and bearings with stiffness and damping. The simulation model shows the division of the three-stage rotor, and the position of the drive end and the nondrive end is shown in Figure 2. The simulation parameters of rotary parts rotors are shown in Table 1. Besides, elastic supports are applied at the front and back rear respectively after the rotor is assembled. The stiffness of the drive end is $8 \times 10^{6} \mathrm{~N} / \mathrm{m}$, and the stiffness of the non-drive end is $1.2 \times 10^{7} \mathrm{~N} / \mathrm{m}$.

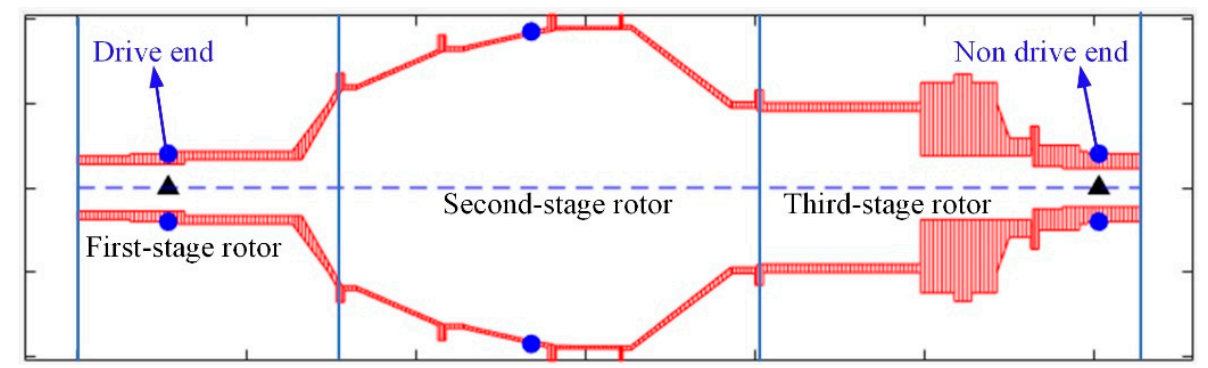

Figure 2. Simulation model of rotor vibration.

Table 1. Simulation parameters of rotary parts rotors.

\begin{tabular}{cccc}
\hline Rotor Parameter & First-Stage Rotor & Second-Stage Rotor & Third-Stage Rotor \\
\hline $\begin{array}{c}\text { Mass weight/g } \\
\text { Height/mm }\end{array}$ & 27,400 & 20,000 & 5800 \\
$\begin{array}{c}\text { Geometric eccentricity } \\
\text { error in X direction/mm }\end{array}$ & 310 & 500 & 330 \\
\hline $\begin{array}{c}\text { Geometric eccentricity } \\
\text { error in Y direction/mm } \\
\text { Geometric eccentricity } \\
\text { error in Z direction/mm } \\
\quad \text { Perpendicularity } / \mathrm{mm}\end{array}$ & 0.001 & 0.001 & 0.001 \\
$\begin{array}{c}\text { Mass eccentricity error in X } \\
\text { direction/mm }\end{array}$ & 0.002 & 0.002 & 0.002 \\
Mass eccentricity error in Y & 0.001 & 0.002 & 0.002 \\
direction/mm & 0.001 & 0.001 & 0.001 \\
Mass eccentricity error in Z & 0.001 & 0.002 & 0.002 \\
direction/mm & 0 & 0.001 & 0.001 \\
Lowest tilt point phase $/{ }^{\circ}$ & 0 & 0.001 & 0.001 \\
\hline
\end{tabular}

Figure 3 shows the simulation results of vibration amplitude at the drive end of the rotor system. The vibration amplitude of the drive end is simulated and analyzed after the assembly of the rotor system under the working speed of the rotary parts. Taking the vibration response of the drive end as an example, the vibration amplitude is $17.55 \mu \mathrm{m}$ when the second and third stage rotors are assembled by the direct assembly strategy. The vibration amplitude is $3.8 \mu \mathrm{m}$ when the second stage rotor assembly phase angle is $170^{\circ}$ and the third stage rotor assembly phase angle is $188^{\circ}$ which is assembled by the optimization assembly strategy. The results show that the vibration amplitude is reduced by $78.3 \%$ through the adjustment of assembly phase. In all, it can be seen that the vibration amplitude of rotary parts rotor system can be changed, and the vibration suppression of rotor system can be realized by adjusting the assembly phase of each stage of the rotor. 


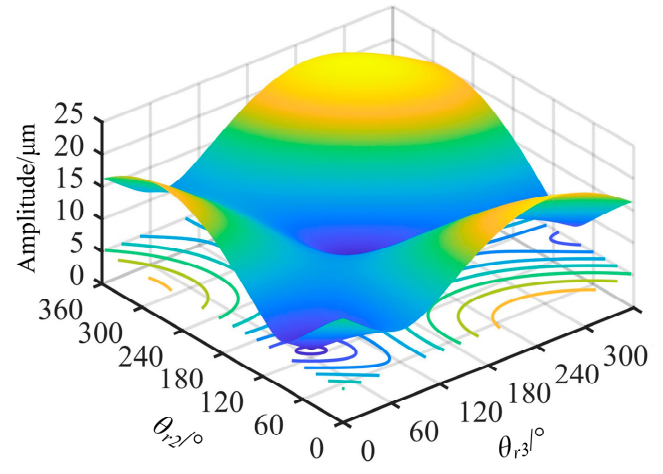

(a)

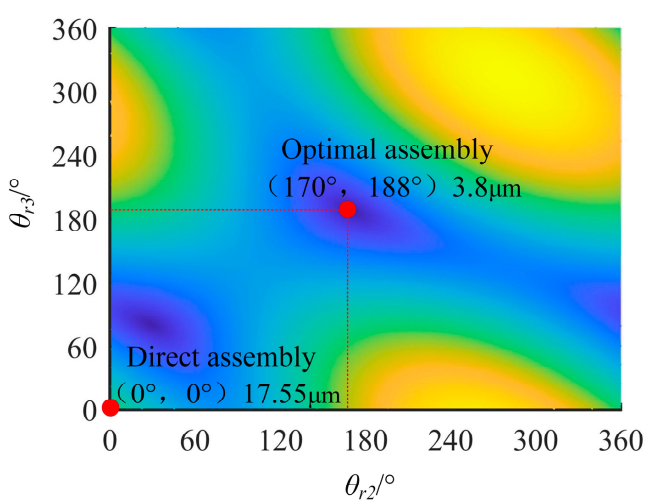

(b)

Figure 3. Vibration amplitude control diagram of drive end under working speed of rotary parts rotor system: (a) Three-dimensional diagram of vibration amplitude changing with assembly phase; (b) Projection diagram of vibration amplitude changing with assembly phase.

To suppress the vibration and make the geometric errors, mass characteristics of the assembled rotary parts rotor meet the eligibility criteria which is obtained from the assembly workshop, this paper carries out three assembly strategies: Direct Assembly, Minimize Vibration Assembly, Optimal Assembly with Double Constraints. Figure 4 shows the results of coaxiality, unbalance, and vibration amplitude under three assembly strategies. It can be seen from Figure 4 that the coaxiality and unbalance of the first assembly method exceeds the eligibility criteria, and the vibration amplitude is the largest of the three assembly methods, which is $17.55 \mu \mathrm{m}$. The unbalance of the second assembly method is lower than the standard line, and the vibration is the smallest of the three assembly methods, but the coaxiality is not constrained, which is far more than the eligibility criteria. The third assembly method constrains the values of coaxiality and unbalance, which meet the eligibility criteria and the vibration amplitude is only $4.3 \mu \mathrm{m}$. Compared with the second assembly method, the third assembly method takes into account both coaxiality and unbalance constraints, which not only realizes the vibration suppression of the rotor, but also ensures that the coaxiality and unbalance of the rotor are within the eligibility criteria after assembly. Therefore, the third assembly strategy should be adopted in the rotary parts assembly process.

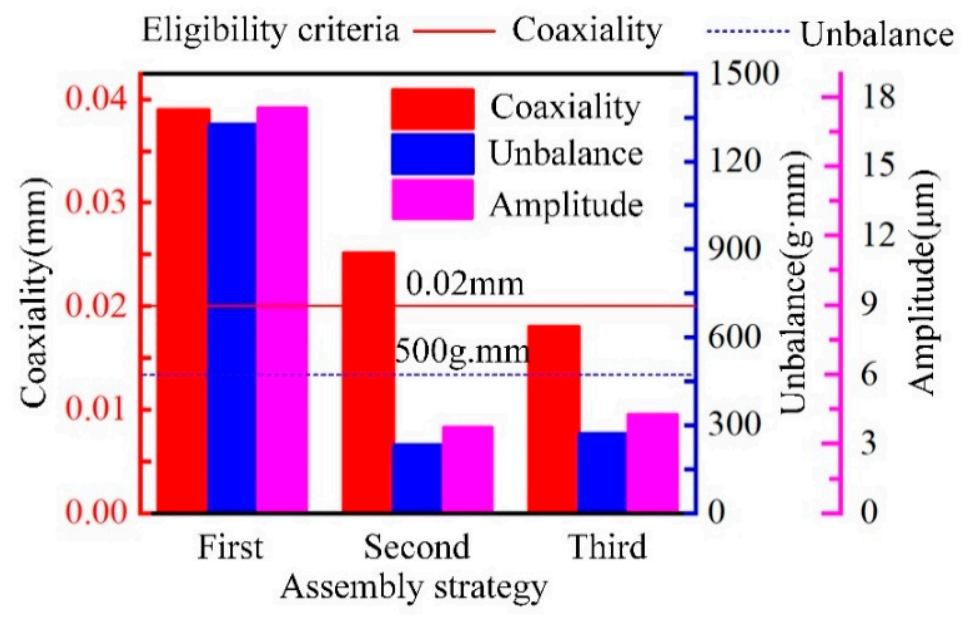

Figure 4. Comparison of vibration amplitude under three assembly models.

The vibration amplitude of the drive end and non-drive end under direct assembly and optimized assembly are shown in Figure 5 when the engine operating speed increases from $0 \mathrm{rpm}$ to $15,000 \mathrm{rpm}$. The direct assembly strategy is not to adjust the assembly phase of all levels of rotor for assembly, and the optimal assembly strategy is the third 
assembly strategy proposed in Section 2.2. Taking the vibration amplitude of drive end as an example which is shown in Figure 5a, the rotor system reaches the first and second critical speed when the speed is $2850 \mathrm{rpm}$ and $5850 \mathrm{rpm}$ and the vibration amplitude of the optimized assembly strategy is obviously lower than that of the direct assembly strategy. The vibration amplitude of the drive end is $3.9 \mu \mathrm{m}$ and $19.6 \mu \mathrm{m}$ respectively under the optimized assembly strategy and the direct assembly strategy when the engine is working at $12,000 \mathrm{rpm}$. The vibration of the engine can be greatly reduced by adjusting the assembly phase of the rotor at all levels. Therefore, the optimized assembly strategy can greatly reduce the high-speed vibration of the engine compared with direct assembly strategy.

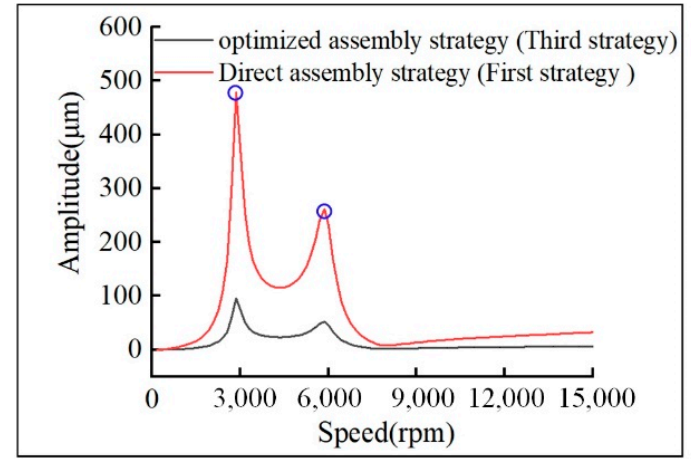

(a)

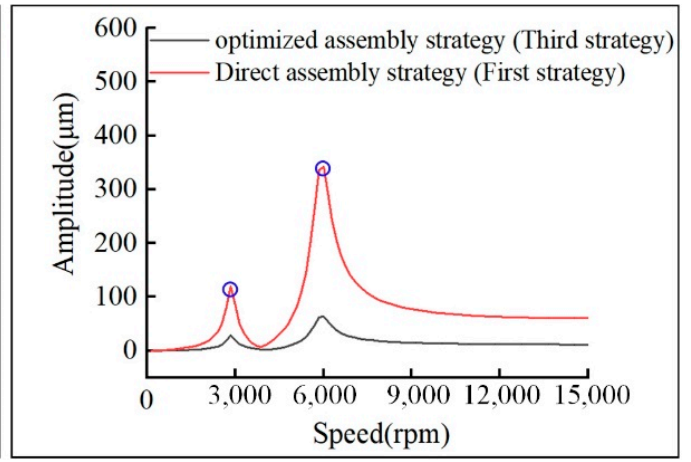

(b)

Figure 5. Vibration amplitude under different assembly strategy: (a) Vibration amplitude of drive end; (b) Vibration amplitude of non-drive end.

\section{Experiment}

\subsection{Parameters Measurement of Rotary Parts Rotors}

Before rotary parts rotors are assembled, it is necessary to measure the geometric error parameters and mass characteristics parameters of each single-stage rotor. The geometric error parameters measurement device is shown in Figure 6a, including precision air bearing turntable, centering and tilt adjustment table, precision chuck and four inductive sensors. Among them, the rotary accuracy of air bearing turntable is less than $0.1 \mu \mathrm{m}$, which provides the measurement reference for the engine rotor. The axial resolution of the centering and tilting adjustment table is $0.2^{\prime \prime}$ and the radial resolution is $0.2 \mu \mathrm{m}$, which can reduce the positioning error between the rotor coordinate system and the turntable coordinate system. The resolution of the four inductive sensors is $0.1 \mu \mathrm{m}$ and the measurement range of sensor- 1 , sensor- 2 is $\pm 0.3 \mathrm{~mm}$, the measurement range of sensor3 , sensor- 4 is $\pm 1.0 \mathrm{~mm}$. The geometric radial error parameters of the bottom and top assembly surfaces are measured by using sensor- 1 and sensor- 2 and the geometric axial error parameters of the bottom and top assembly surfaces are measured by using sensor-3 and sensor-4, respectively.

The specific measurement process is as follows: (1) start the air bearing turntable until it rotates at a stable speed, then adjust the sensor- 1 and sensor- 3 probes to make them contact with the radial and axial reference surface to measure the radial and axial geometric errors of bottom face of each rotor, (2) adjust the centering and tilt adjustment table to ensure that the geometric axis of the rotor coincides with the rotation axis, (3) adjust the sensor-2 and sensor-4 probes to make them contact with the radial and axial top surface of the rotor to measure the radial and axial geometric errors of top face. The specific geometric characteristic parameters of each stage rotor are shown in Table 2. 


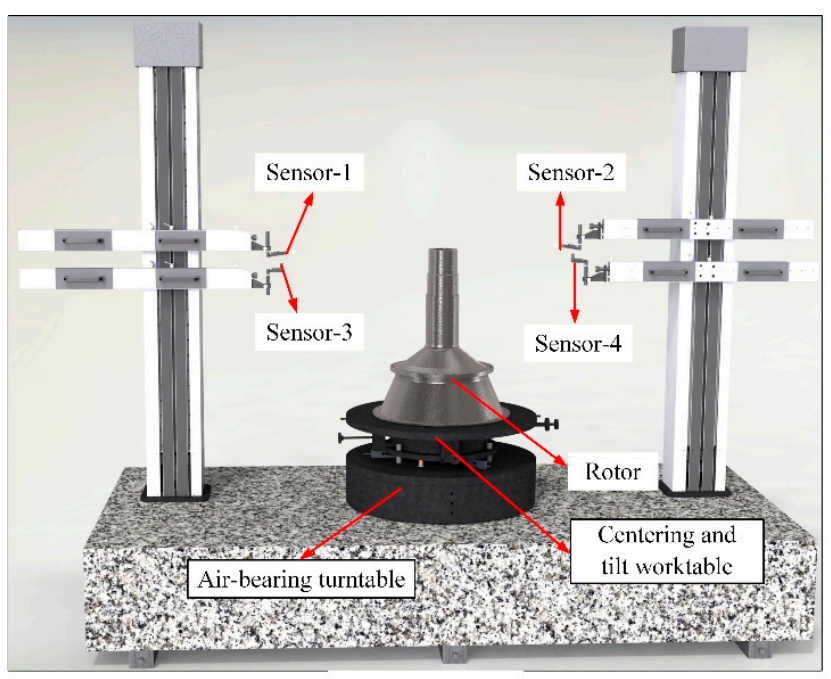

(a)

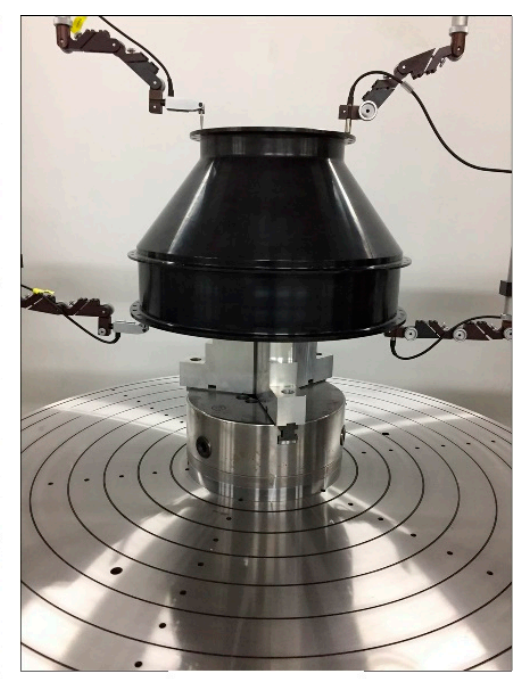

(b)

Figure 6. Test device diagram of rotor geometric parameters and mass parameters: (a) Precision air bearing turntable; (b) Experimental rotor.

Table 2. Rotor geometric characteristics and parameter errors of rotary parts rotors.

\begin{tabular}{cccc}
\hline Rotor Parameter & First-Stage Rotor & Second-Stage Rotor & Third-Stage Rotor \\
\hline Radius of top face/mm & 43 & 189 & 100 \\
Radius of bottom face & 189 & 100 & 66 \\
/mm & 310 & 500 & 330 \\
Height/mm & 0.0026 & 0.0011 & -0.0119 \\
Geometric eccentricity & & & -0.0047 \\
error in X direction/mm & 0.0419 & 0.0051 & 0.0003 \\
$\quad \begin{array}{l}\text { Geometric eccentricity } \\
\text { error in Y direction/mm }\end{array}$ & & 0.0015 & 0.9 \\
$\quad$ Geometric eccentricity & 0.0021 & 3.1 & 51 \\
error in Z direction/mm & 2.3 & 221 & \\
Tilt angle/" & 220 & &
\end{tabular}

We can obtain the initial unbalance and the corresponding angle of the single-stage rotor by using the vertical balancing machine. Each stage rotor mass centroid eccentricity error parameters that can be calculated by their own initial unbalance and the corresponding angle, as shown in Table 3.

Table 3. Mass characteristics and centroid parameter errors of rotary parts rotors.

\begin{tabular}{cccc}
\hline Rotor Parameter & First-Stage Rotor & Second-Stage Rotor & Third-Stage Rotor \\
\hline Mass weight/g & 27,850 & 19,278 & 53,278 \\
Mass eccentricity error in & 0.0042 & -0.0080 & -0.0042 \\
$\quad$ X direction/mm & & -0.0067 & 0.0041 \\
Mass eccentricity error in & -0.0039 & & \\
Y direction/mm & & & \\
\hline
\end{tabular}

\subsection{Vibration Test}

The vibration test device of rotary parts is shown in Figure 7, including vibration isolation platform, power system, experimental rotors, and acceleration sensors, etc. The power system provides the power source and the required speed of the rotor rotation. The acceleration sensors are arranged in two orthogonal directions of the rotor to measure the vibration amplitude. The experimental rotors are assembled by the direct assembly strategy and the double constraint optimization assembly strategy for vibration measurement. 


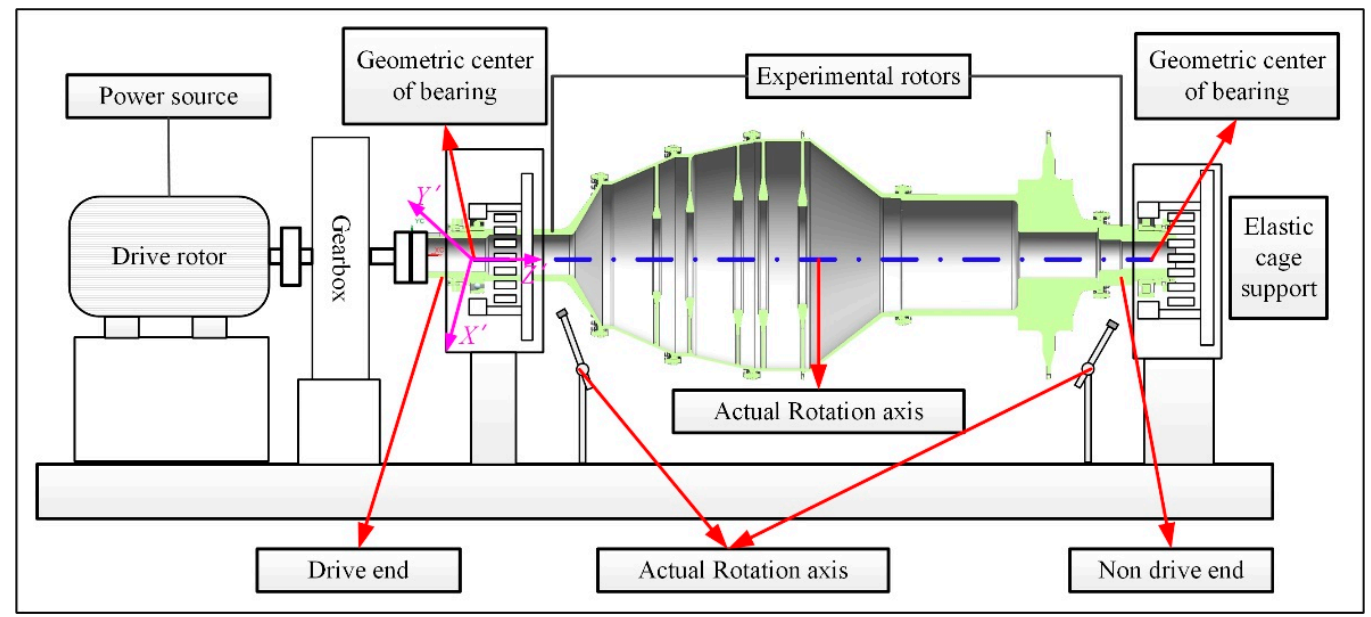

Figure 7. Rotary parts vibration test device.

According to Equation (9), the coordinate of the rotor center and the mass centroid can be obtained from the parameter as shown in Tables 2 and 3. The rotor coaxiality and unbalance assembled by First assembly strategy and Third assembly strategy are shown in Table 4.

Table 4. Coaxiality and unbalance under different assembly strategy.

\begin{tabular}{ccccc}
\hline \multirow{2}{*}{ Rotor Parameter } & \multicolumn{2}{c}{ Coaxiality/mm } & \multicolumn{2}{c}{ Unbalance/g.mm } \\
\cline { 2 - 5 } & $\begin{array}{c}\text { First Assem-Bly } \\
\text { Strategy }\end{array}$ & $\begin{array}{c}\text { Third Assembly } \\
\text { Strategy }\end{array}$ & $\begin{array}{c}\text { First Assem-Bly } \\
\text { Strategy }\end{array}$ & $\begin{array}{c}\text { Third Assembly } \\
\text { Strategy }\end{array}$ \\
\hline $\begin{array}{c}\text { First-stage rotor } \\
\text { Second-stage } \\
\text { rotor }\end{array}$ & 0.012 & 0.012 & 84.45 & 84.45 \\
Third-stage rotor & 0.068 & 0.030 & 810.41 & 532.65 \\
\hline
\end{tabular}

The eligibility criteria of coaxiality is less than or equal to $0.05 \mathrm{~mm}$ and the eligibility criteria of unbalance is less than or equal to $800 \mathrm{~g} \cdot \mathrm{mm}$. It can be seen from Table 4 that the coaxiality and unbalance by the optimized assembly strategy are better than those by the direct assembly strategy. The coaxiality and unbalance by the third assembly strategy is the only one which can meet the assembly requirements. With the increase of rotor stages, the advantages of the optimized assembly strategy are more obvious. Taking the three-stage rotor as an example, the coaxiality by the third assembly strategy is reduced by $44.1 \%$, and the unbalance is reduced by $78.4 \%$, which shows the advantages of the third assembly strategy.

The vibration amplitude of the rotary parts rotors which are assembled by the optimized assembly strategy is compared with the vibration amplitude assembled by the direct assembly strategy is shown in Figure 8. The vibration amplitude increases with the increase of the speed and then the amplitude of the rotor begins to decrease when the speed reaches the critical speed, which is consistent with the amplitude changing trend shown in Figure 4 and the correctness of the model is verified in this paper. The vibration amplitude of the rotary parts rotors by the third assembly strategy is $3.3 \mu \mathrm{m}$, $3.1 \mu \mathrm{m}, 4.8 \mu \mathrm{m}$, and $3.1 \mu \mathrm{m}$ less than the vibration amplitude using direct assembly strategy when the speed is $4000 \mathrm{rpm}, 5000 \mathrm{rpm}, 6000 \mathrm{rpm}$, and $7000 \mathrm{rpm}$, respectively. The average amplitude is reduced by $22.5 \%$. Therefore, the double constraint optimal assembly strategy established in this paper can effectively suppress the rotary parts rotor vibration. We did five sets of repetitive experiments, when the speed is $4000 \mathrm{rpm}$, the vibration amplitudes of optimized assembly strategy are $8 \mu \mathrm{m}, 8.7 \mu \mathrm{m}, 7.1 \mu \mathrm{m}, 6.4 \mu \mathrm{m}$, and $6.8 \mu \mathrm{m}$. The mean is $7.4 \mu \mathrm{m}$, the standard deviation is $0.84 \mu \mathrm{m}$, the uncertainty introduced by measurement repeatability $u\left(x_{1}\right)=0.84 \mu \mathrm{m}$, the uncertainty introduced by the resolution of eddy current 
sensor $u\left(x_{2}\right)=0.01 \mu \mathrm{m}$, the uncertainty introduced by the errors of eddy current sensor $u\left(x_{3}\right)=0.28 \mu \mathrm{m}$, the standard uncertainty is $U=2.10 \mu \mathrm{m}(k=2)$.

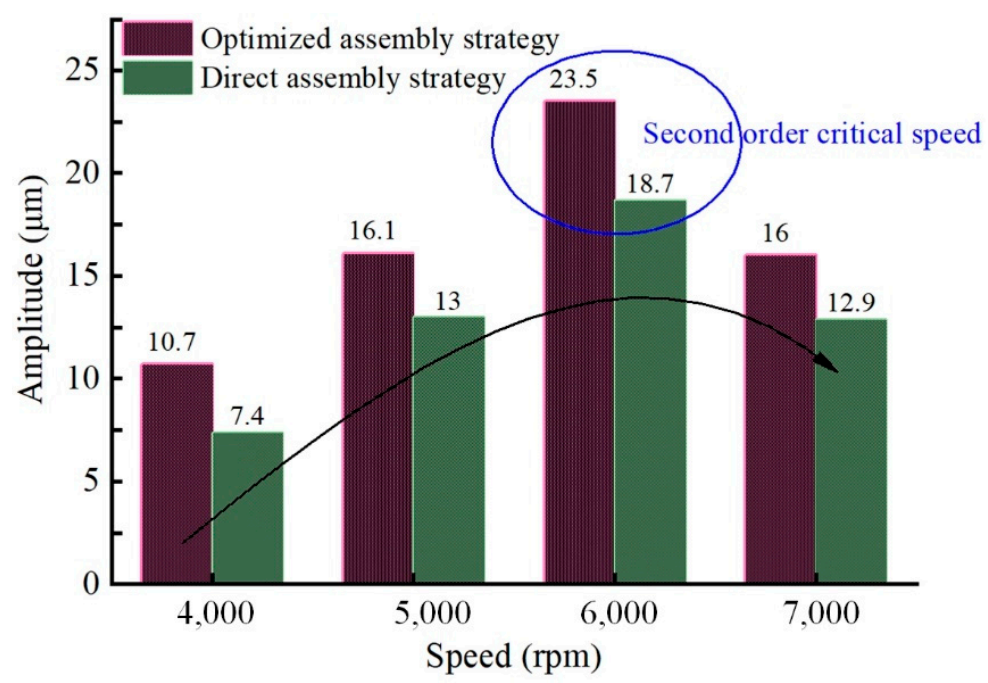

Figure 8. Vibration amplitude of rotary parts rotor under different assembly strategies.

\section{Conclusions}

A single-stage rotor centroid transformation model is established based on the actual rotating axis coordinate system of rotary parts in this paper, and the conclusions are as following through simulation analysis and experimental verification:

This paper proposed a method to reduce vibration by adjusting the rotor assembly phase. Firstly, the rotor stacking mass centroid transformation model in the rotating axis coordinate system is established, and then the dynamic analysis model of rotary parts vibration response based on the centroid stack is studied. Results show that the vibration amplitude of the rotor is affected and can be reduced by adjustment of the assembly phase of each rotor. The vibration amplitude of the engine is reduced by $22.5 \%$ on average when using optimal assembly phase of the multi-stage rotors obtained by the third assembly model. Besides, the coaxiality and the unbalance are reduced by $44.1 \%$ and $78.4 \%$, which shows the advantages of the third assembly strategy.

In this paper, the mechanism of unbalance exciting force and vibration response caused by rotor centroid offset is studied, so as to realize the vibration suppression of rotor. In future work, to realize the effective prediction and suppression of engine rotor vibration, we may study the influence of interaction between other exciting forces and vibration.

Author Contributions: Conceptualization, Y.L.; methodology R.L.; Software and Validation, Z.C., C.L., C.S. and Y.M.; Investigation, P.X., X.W. All authors have read and agreed to the published version of the manuscript.

Funding: This research was funded by the National Natural Science Foundation major research projects of China (grant number 91960109), the National Natural Science Foundation of China (grant number 51805117), the China postal Postdoctoral Science Foundation (grant number 2019M651279), the Heilongjiang Postdoctoral Fund (grant number LBH-Z18078).

Institutional Review Board Statement: Not applicable.

Informed Consent Statement: Not applicable.

Data Availability Statement: The data presented in this study are available on request from the corresponding author. The data are not publicly available due to the data confidentiality related to the fund.

Acknowledgments: This work was supported by the National Natural Science Foundation major research projects of China (grant number 91960109), the National Natural Science Foundation of 
China (grant number 51805117), the China postal Postdoctoral Science Foundation (grant number 2019M651279), the Heilongiiang Postdoctoral Fund (grant number LBH-Z18078).

Conflicts of Interest: The authors declare no conflict of interest.

\section{References}

1. Yongcun, C.; Sier, D.; Wenhu, Z.; Guoding, C. The impact of roller dynamic unbalance of high-speed cylindrical roller bearing on the cage nonlinear dynamic characteristics. Mech. Mach. Theory 2017, 118, 65-83. [CrossRef]

2. Cui, Y.; Deng, S.; Niu, R.; Chen, G. Vibration effect analysis of roller dynamic unbalance on the cage of high-speed cylindrical roller bearing. J. Sound Vibr. 2018, 434, 314-335. [CrossRef]

3. Wang, D.; Wang, N.; Chen, K. Unbalance response of a magnetic suspended dual-rotor system. Proc. Inst. Mech. Eng. Part. G J. Aerosp. Eng. 2019, 233, 5758-5772. [CrossRef]

4. Wang, H.; Gong, J.; Chen, G. Characteristics analysis of aero-engine whole vibration response with rolling bearing radial clearance. J. Mech. Sci. Technol. 2017, 31, 2129-2141. [CrossRef]

5. Liu, Y.; Zhang, M.; Sun, C.; Hu, M.; Chen, D.; Liu, Z.; Tan, J. A method to minimize stage-by-stage initial unbalance in the aero engine assembly of multistage rotors. Aerosp. Sci. Technol. 2019, 85, 270-276. [CrossRef]

6. Hussain, T.; Yang, Z.; Popov, A.A.; Mcwilliam, S. Straight-Build Assembly Optimization: A Method to Minimize Stage-by-Stage Eccentricity Error in the Assembly of Axisymmetric Rigid Components (Two-Dimensional Case Study). J. Manuf. Sci. Eng.-Trans. 2011, 133, 031014. [CrossRef]

7. Yang, Z.; Mcwilliam, S.; Popov, A.A.; Hussain, T. A probabilistic approach to variation propagation control for straight build in mechanical assembly. J. Mech. Sci. Technol. 2013, 64, 1029-1047. [CrossRef]

8. Lei, W.; Sun, C.; Tan, J.; Bo, Z.; Gu, W. Improvement of location and orientation tolerances propagation control in cylindrical components assembly using stack-build assembly technique. Assem. Autom. 2015, 35, 358-366. [CrossRef]

9. Rao, S.S.; Wu, A. Optimum tolerance allocation in mechanical assemblies using an interval method. Eng. Optim. 2005, 37, 237-257. [CrossRef]

10. Yang, Z.; Mcwilliam, S.; Popov, A.A.; Hussain, T.; Yang, H. Dimensional variation propagation analysis in straight-build mechanical assemblies using a probabilistic approach. J. Manuf. Syst. 2013, 32, 348-356. [CrossRef]

11. Yang, Z.; Popov, A.A.; Mcwilliam, S. Variation propagation control in mechanical assembly of cylindrical components. J. Manuf. Syst. 2012, 31, 162-176. [CrossRef]

12. Sun, C.; Wang, L.; Tan, J.; Zhao, B.; Tang, Y. Design of roundness measurement model with multi-systematic error for cylindrical components with large radius. Rev. Sci. Instrum. 2016, 87, 025110. [CrossRef]

13. Liu, J.; Fa-Yong, W.U.; Wang, J. Optimization Technique of Aeroengine Rotor Assembly. Aeroengine 2014, 40, 75-78. [CrossRef]

14. Maldonado, D.; Karev, A.; Hagedorn, P.; Ritto, T.G.; Sampaio, R. Analysis of a rotor dynamic system with anisotropy and nonlinearity using the Floquet theory and the method of normal forms. J. Sound Vibr. 2019, 453, 201-213. [CrossRef]

15. Eissa, M.; Saeed, N.A. Nonlinear vibration control of a horizontally supported Jeffcott-rotor system. J. Vib. Control. 2018, 24, 5898-5921. [CrossRef]

16. Xu, L.X. A general method for impact dynamic analysis of a planar multi-body system with a rolling ball bearing joint. Nonlinear Dyn. 2014, 78, 857-879. [CrossRef]

17. Lees, A.W.; Sinha, J.K.; Friswell, M.I. Model-based identification of rotating machines. Mech. Syst. Signal. Proc. 2009, 23, 1884-1893. [CrossRef]

18. Zhou, S.; Shi, J. Imbalance Estimation for Speed-Varying Rigid Rotors Using Time-Varying Observer. J. Dyn. Syst. Meas. Control Trans. ASME 2001, 123, 637-644. [CrossRef]

19. Yao, J.; Liu, L.; Yang, F.; Scarpa, F.; Gao, J. Identification and optimization of unbalance parameters in rotor-bearing systems. J. Sound Vibr. 2018, 431, 54-69. [CrossRef]

20. Chen, G. Vibration modelling and verifications for whole aero-engine. J. Sound Vibr. 2015, 349, 163-176. [CrossRef]

21. Breńkacz, Ł.; Bagiński, P.; Korbicz, K.J. Vibration damping of the anti-vibration platform intended for use in combination with audio/music devices. J. Vibr. Eng. 2020, 22, 578-593. [CrossRef]

22. Breńkacz, Ł.; Bagiński, P.; Żywica, G. Experimental Research on Foil Vibrations in a Gas Foil Bearing Carried Out Using an Ultra-High-Speed Camera. Appl. Sci. 2021, 11, 878. [CrossRef]

23. Yao, H.; Cao, Y.; Ding, Z.; Wen, B. Using grounded nonlinear energy sinks to suppress lateral vibration in rotor systems. Mech. Syst. Signal. Proc. 2019, 124, 237-253. [CrossRef]

24. Qiu, Y.; Jiang, S. Suppression of low-frequency vibration for rotor-bearing system of flywheel energy storage system. Mech. Syst. Signal. Proc. 2019, 121, 496-508. [CrossRef]

25. Lusty, C.; Bailey, N.Y.; Keogh, P.S. Control of Flexible Rotor Vibration with Flexibly Mounted Active Magnetic Bearings. IEEEASME Trans. Mechatron. 2018, 23, 2870-2880. [CrossRef]

26. Sun, C.; Liu, Z.; Liu, Y.; Wang, X.; Tan, J. An Adjustment Method of Geometry and Mass Centers for Precision Rotors Assembly. IEEE Access 2019, 7, 169992-170002. [CrossRef] 